\title{
About the "Mixture" of Discourses in the Use of Mathematics in Signal Theory À propos du «mélange» de discours dans l'utilisation des mathématiques en théorie du signal
}

\author{
Reinhard Hochmuth ${ }^{1}$ \\ Institute for Didactics of Mathematics and Physics, Leibniz Universität Hannover, \\ Germany \\ https://orcid.org/0000-0002-4041-8706 \\ Jana Peters ${ }^{2}$ \\ Institute for Didactics of Mathematics and Physics, Leibniz Universität Hannover, \\ Germany \\ https://orcid.org/0000-0003-0628-7105
}

\begin{abstract}
An important issue for research in university mathematics education is the use of mathematics in engineering. Here we focus on praxeologies in a course on system and signal theory (SST), which represents a typical module in electrical engineering studies in the third or fourth semester. In such courses, mathematics already studied in introductory mathematics courses will be applied, but also enriched by the introduction and development of new practices, in particular the so-called Dirac-impulse. We claim that the introduction and justification of the Dirac-impulse in SST is a convenient case where basic facets of epistemological relations between mathematics and engineering sciences might be illustrated and shown to be important for a detailed description and analysis of logos blocks of praxeologies. The background for our considerations regarding logos blocks of praxeologies that concern the introduction of the Dirac-impulse is given by philosophical studies by Wahsner and Borzeszkowski $(1992,2012)$ and a few illuminating remarks by Dirac.
\end{abstract}

Keywords: Signal Theory, Dirac impulse, Epistemology, ATD.

\footnotetext{
${ }^{1}$ hochmuth@idmp.uni-hannover.de

2 peters@idmp.uni-hannover.de
} 


\section{Résumé}

Une question importante pour la recherche en éducation mathématique universitaire est l'utilisation des mathématiques en ingénierie. Ici, nous nous concentrons sur les praxéologies dans un cours sur la théorie du système et du signal (SST), qui représente un module typique dans les études d'ingénierie électrique au troisième ou quatrième semestre. Dans ces cours, non seulement applique-t-on les mathématiques déjà enseignées et apprises dans les cours d'introduction à la mathématique, mais on introduit et utilise aussi de nouveaux concepts mathématiques, en particulier ce que l'on appelle l'impulsion de Dirac. Nous affirmons que l'introduction et la justification de l'impulsion de Dirac dans SST est un cas pratique par lequel les facettes fondamentales des relations épistémologiques entre mathématiques et ingénierie pourraient être illustrées et démontrées importantes pour la description détaillée et l'analyse des logos blocs de praxéologies. Le contexte de nos considérations au sujet des logos blocs de praxéologies concernant l'introduction de l'impulsion de Dirac est donné par des études philosophiques de Wahsner et Borzeszkowski (1992, 2012) et quelques remarques éclairantes de Dirac. Mots-clés: Théorie du signal, impulsion de Dirac, épistémologie, TAD. 


\section{About the "Mixture" of Discourses in the Use of Mathematics in Signal Theory}

The use of mathematics in engineering and sciences is an important topic for research in university mathematics education. This is partly because of high dropout rates and the search for measures optimizing teaching and learning of mathematics in other study fields. Here we focus on praxeologies in a course on system and signal theory (SST) representing a typical module in electrical engineering studies in the third or fourth semester.

In recent years several papers have analyzed mathematical practices in engineering. Generally there are two interrelated foci: The first one is on aspects of modelling and application problems, where typically an engineering problem is prepared such that mathematics from introductory higher mathematics courses has to be applied to solve the task. In most cases it is obvious that the modelling cycle used for school mathematics, which separates the world in a mathematical world and the rest of the world (see for example Blum \& Leiss (2005)), is not appropriate for describing and analyzing such activities since the engineering problem is a priori formulated in mathematical terms. Therefore, it has been suggested to use ATD for describing and analyzing the intertwined mathematical and engineering practices (see for example HOCHMUTH, BIEHLER AND SCHREIBER, 2014)). Moreover, Castela and Romo (2011) have introduced extended praxeological models, an idea which was adapted by Peters, Hochmuth \& Schreiber (2017) to analyze tasks in a signal and system theory course. The institutional separation between mathematics and engineering in courses and curricula were also the starting point for investigations in (Barquero, Serrano, L. \& Serrano V., 2013), where so called "study and research courses" are proposed for overcoming the dominant epistemology of "applicationism". Our research connects in particular the observations by Barquero, Bosch \& Gascón (2011) regarding the "distinction between mathematics and the rest of 
natural sciences" and contributes to another application of the scale of level of codeterminations (Bosch \& Gascón, 2006) studying conditions that frame the use of mathematics in other sciences.

The second focus is on the use of symbols: symbols are often both representations of mathematical variables and representations of physical or engineering quantities. It is often not clear to novices how they have to interpret symbols in view of a task and which argumentations are required or forbidden, see for example (Tuminaro \& Redish, 2007; Hochmuth \& Schreiber, 2015; Alpers, 2017; Peters, Hochmuth \& Schreiber, 2017).

Here we adopt a slightly different position: We relate the intertwining of mathematical and engineering ideas to its historical genesis process and the dissolving of certain fundamental epistemological problems. Often it does not seem to be important for an understanding of actual teaching and learning contexts to enlighten such issues in detail. In our praxeological analyses of the use of mathematics in SST we came across those issues, as we tried to substantiate technological and theoretical issues: In the analysis of text-books besides clear arguments that are based on techniques and technologies developed in higher mathematics or electrical engineering, we observed vague argumentations bobbing up at certain steps. We had the impression, that the vague steps arise at points that are significant both for an understanding what it means that an engineering practice is pragmatic and for a better understanding of switching between mathematics and engineering.

Therefore, we began to think about incorporating basic observations from (Wahsner \& Borzeszkowski, 1992; Borzeszkowski \& Wahsner, 2012) that take into account the relation between mathematics and physics. They raise several epistemological issues which have to be resolved in any mathematically based theory intending to describe and calculate "nature". These epistemological issues are also 
relevant for engineering sciences, since they can be interpreted in relation to those issues as concretizations in view of subject related aims embedded within culture-historical as well as socio-economical processes.

After clarifying the mathematical context and focus of our paper as well as the theoretical framework in sections 2 and 3, we exemplarily investigate passages from a SST text-book introducing the Dirac-impulse. A sketchy praxeological analysis allows linking vague passages to fundamental epistemological issues concerning the relation between mathematics and physics, respectively engineering sciences. We support our observations by a few illuminating remarks by Dirac.

\section{Context and focus}

We analyze the introduction of the Dirac-impulse in (Fettweis, 1996) with a focus on specific steps in the justification of some of its characterizing properties:

$$
\delta(t)=\left\{\begin{array}{l}
\infty \text { for } t=0 \\
0 \text { for } t \neq 0
\end{array}, \int_{-\infty}^{\infty} \delta(t) d t=1, \int_{-\infty}^{\infty} \varphi(t) \delta\left(t-t_{0}\right)=\right.
$$

These properties are typically also introduced and used in quantum mechanics and go back to Dirac (1927), who was already aware of the problem that $\delta$ could not be a "normal" function and has to be interpreted in a specific way:

Strictly, of course, $\delta(\mathrm{x})$ is not a proper function of $\mathrm{x}$, but can be regarded only as a limit of a certain sequence of functions. All the same one can use $\delta(x)$ as though it were a proper function for practically all the purposes of quantum mechanics without getting incorrect results. (p. 625)

The mathematical knowledge of that time did not provide a consistent and welldefined framework for the Dirac-impulse, which was, by good reasons, not a real problem for Dirac, Heisenberg and Pauli in contrast to, e.g., von Neumann (Peters, 2004). Nowadays there are several possibilities to introduce the Dirac-impulse respecting the actual socio-mathematical norms in mathematics as a science. We remind of the following three possibilities: 
a) In Functional analysis (see for example Schwartz (1947)) $\delta$ is considered as a distribution, that is a linear and continuous functional on so called test function spaces like $C_{0}^{\infty}(\mathbb{R})$ or $S(\mathbb{R})$.

b) In Non-standard analysis (see for example Landers \& Rogge (2013)) $\delta$ can be seen as a "normal" function from the hyperreal numbers ${ }^{*} \mathbb{R}$ to ${ }^{*} \mathbb{R}$. In the 19th and beginning 20th century there were some discussions (Purkert, 1990) about the usefulness of the $\varepsilon$ - $\delta$-calculus for engineering students and it was proposed, for example by Weisbach (1860), to teach instead Leibniz's infinitesimal calculus, which can be seen as a predecessor of non-standard analysis. Nowadays, non-standard analysis is typically not taught in mathematic courses for engineers in Germany.

c) Another possibility, which is partly adopted in (Fettweis, 1996), considers distributions as limits of sequences of functions, which converge in a specific way (Antosik, Mikusiński, \& Sikorski, 1973). This approach can be elaborated on a level that is the most part compatible with higher mathematics taught in courses for engineers. Within this framework, integrals with respect to $\delta$ were introduced and interpreted in a symbolic way, as notions representing the result of limit processes.

Obviously the presentation in Fettweis is mathematically not complete and it could be argued whether and how it could be supplemented. In the following we do not want to discuss whether the introduction of more complete and formal mathematics would be useful from an engineering point of view. Instead we intend to demonstrate that certain appearing gaps can be linked to fundamental epistemological issues concerning the relation between mathematics and physics. This suggests that the gaps and their character 
are in the first instance not the deficit result of too little mathematics but the expression of a specific historic and institutional resolution of certain epistemological issues.

\section{Theoretical framework}

We combine a praxeological analysis with conclusions from historicphilosophical considerations based on a dialectic and materialistic point of view concerning the relation between mathematics and physics by Wahsner and Borzeszkowski $(1992,2012)$. We believe that those conclusions refer also to inherent characteristics of the relation between mathematics and engineering sciences. According to the status of our work in progress we use the praxeological approach for reconstructing the engineering content and inject philosophical considerations in the analyses of the logos-block focusing on the relation between "mathematics" (distribution theory) and "physical reality" (signals).

\section{Anthropological theory of the didactic}

In our analysis we address two concepts of ATD: First we outline a praxeological analysis of a SST-practice, where we focus the most elementary model of praxis/logos blocks. This praxeological model consists of the praxis block $\mathrm{P}$ containing tasks and techniques used to solve them and the logos block L containing the technological and theoretical discourse describing justifications, explanations and production of the elements of the praxis-block. This P/L-model could be refined into the so called 4T-model where the praxis block is differentiated into tasks $\mathrm{T}$ and techniques $\tau$ and the logos block is differentiated into technology $\theta$ and theory $\Theta$, where theory forms a discourse on technology that is more elaborated and abstract (Chevallard, Bosch, \& Kim, 2015). We forgo formulating tasks and techniques as well as technology and theory in detail because of limited space and since these details seem not necessary for representing the main point 
of this paper. Elements of the praxis block $\mathrm{P}$, will be denoted by $p_{i}$, and elements of the $\operatorname{logos}$ block L, by $l_{i}$.

Second, for a more detailed understanding of technological-theoretical facets, that form the logos block, we give a rough allocation to higher levels of codetermination.

\section{Epistemological-philosophical observations regarding physics}

In philosophical and concrete historical studies, Wahsner and Borzeszkowski $(1992,2012)$ figure out those conceptual and experimental-objective preparations within physics that facilitate to use mathematics as mean for expressing, describing and analyzing dynamics in terms of laws and to link mathematics with measuring practices. The following both aspects are in particular important (Wahsner \& Borzeszkowski, 1992, pp. 125-135):

a) Since only finite distances are measurable, conceptual contradictory identifications of infinite or infinitesimal quantities, which arise in mathematical structures, with finite quantities are enforced. The particular context dependent adequate but from a mathematical perspective inconsistent use of mathematical concepts is historically one of the most original achievements of physics.

b) Only effects of properties of objects are measurable and not dynamic interactional relations. This leads to the question, which behavior can be transformed to a property. Related answers could be found studying the complicated historical genesis of physical measured quantities.

Physical quantities are thinking-objects, which are constructed on the basis of real equalities, checked by specific instruments in specific experiments; they are tools for investigating real objects in contexts. Considering and treating physical quantities under 
the measurement aspect allows to formulate dynamics related laws in such a way that their assertions can empirically be proved.

In contrast to physics, quantities appear in mathematics merely within functional structured systems that presuppose their existence. This facilitates mathematics to be without inherent contradictions and formally consistent, but, at the same time, disable mathematics to make assertions about real objects and their behavior. Therefore mathematics needs physics (or another empirical science) to make statements about reality (p. 128). On the other hand, physics needs mathematics for measurements, calculations and expressing dynamic interactions by laws, that is: mathematics allows making basic relations calculable and measurable.

\section{Praxeological analysis}

In this section we present an ATD analysis of the introduction of the Deltaimpulse in (FETTWEIS, 1996). The main ideas and results of this analysis are also relevant for related SST-books like Girod, Rabenstein and Stenger (2007).

\section{General considerations on signals}

Fettweis characterizes signal and system theory not as a technical but as a, in general, physical discipline. The author stresses the importance of physical understanding and argues that an increasing elaboration of mathematical concepts would not only go beyond the scope of the book, but would make the understanding of the physical reasoning of methodological issues increasingly harder. He constructs the relation between mathematics and physics as a dilemma between mathematical precision and the understanding of physical reasoning. This positioning between mathematical precision 
and the understanding of physical reasoning affects the praxeologies, especially their logos-blocks.

The focus on physical understanding leads Fettweis in particular to a general principle concerning two different but connected concepts of signals: "real signals $\mathrm{x}(\mathrm{t})$ " occur at communications transmission and are irregular. Furthermore, they have the following properties:

They are of finite duration, i.e. there exist $t_{0}$ and $t_{1}$ with $t_{1}>t_{0}$, such that $x(t)=0$ for $\mathrm{t}<t_{0}$ and $\mathrm{t}>t_{1}$.

They are continuous for all $t \in(-\infty, \infty)$.

They are sufficiently differentiable.

Following Fettweis (p. 5) real signals are characterized by irregularity and high diversity, so they are inappropriate for numerical and analytical calculations and are not usable as measurement signals. Therefore "idealized signals", which will unavoidably violate some of the properties (1) to (3), are introduced. In spite of their simplicity, using idealized signals can cause difficulties, especially with respect to convergence. As an example, Fettweis (pp. 8) considers the unit step function that satisfies none of the properties (1) to (3)

$$
u(t)=\left\{\begin{array}{c}
0 \text { for } t<0 \\
1 / 2 \text { for } t=0 \\
1 \text { for } t>0
\end{array}\right.
$$

In such a case the idealized signal can be replaced by real signals, such that the specific difficulty does not arise any more. After an analysis using real signals the replacement can be reversed. This general principle is illustrated in Figure 1:

Figure 1

Illustration of the interplay between idealized and real signals

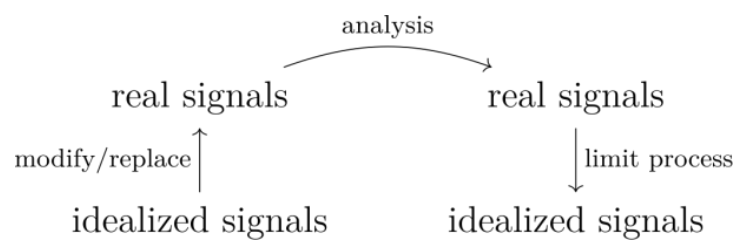


The unit step function $u(t)$, for example, could be replaced by continuous, in $\mathrm{t}=0$ rapidly increasing real signals $f_{n}(t)$, c.f. Figure 2 .

Figure 2

Function series approximating the unit step function

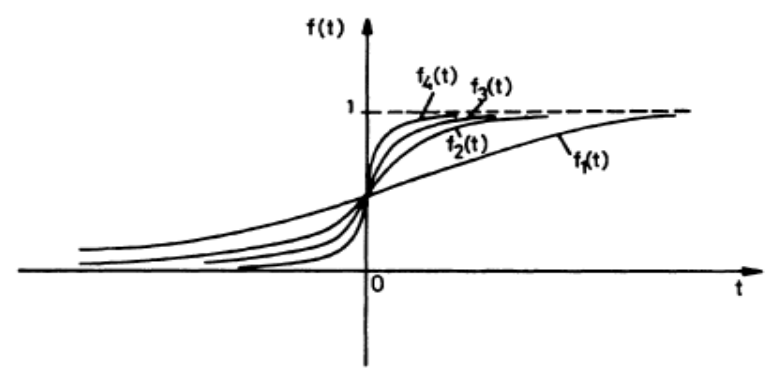

The approximation is symbolically expressed by $u(t)=\left\{f_{n}(t)\right\}$ and also written as limit process: $u(t)=\lim _{n \rightarrow \infty} f_{n}(t)$.

The general principle is justified by referencing the compliance with approaches in other physical disciplines (p.6), by a need of physics to use function series for approximations and by claiming that an adequate application of the principle generates unambiguous and correct results (p. 12). In the same paragraph Fettweis refers also to Distribution Theory as a mathematical domain. He explicitly refers the work of L. Schwartz, that is presented in a "very abstract and physically less appealing form" (p. 12) and the work of Antosik, Mikusiński, and Sikorski (1973), that could be seen as an elaborated mathematical basis for the presentation in Fettweis.

\section{The Delta-impulse}

In this section we provide a sketchy praxeological analysis of the introduction of the Delta-impulse. First we summarize elements of the praxis blocks $p_{i}$. Then we describe the technological-theoretical discourse with regard to the considerations in 4.1. We specify the elements of the logos block by $l_{i}$. 
The idealized impulse $\delta(t)$ is defined by a series of real impulse-functions $\left(p_{1}\right)$ : $\delta(t)=\left\{f_{n}(t)\right\}$ with the following properties

(1) $f_{n}(t)$ has width $2 \epsilon_{n}$ with $\lim _{n \rightarrow \infty} \epsilon_{n}=0$.

(2) All impulses $f_{n}$ have the same normalized area: $\int_{-\infty}^{\infty} f_{n}(t) d t=1$

This definition is illustrated by Figure 3:

Figure 3

Function series representing the Delta-impulse

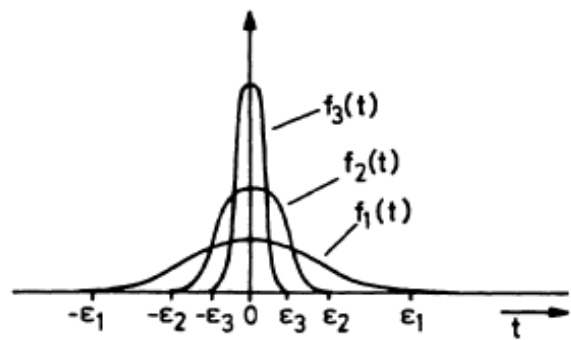

Illustrating properties of functions by graphical representations $\left(p_{2}\right)$ is a common practice in engineering textbooks and in particular in SST. By idealizing (i) and (ii) the two properties a) $\delta(t)=\left\{\begin{array}{c}\infty \text { for } t=0 \\ 0 \text { for } t \neq 0\end{array}\right.$ and b) $\int_{-\infty}^{\infty} \delta(t) d t=1$ are assigned $\left(p_{3}\right)$ and the idealized impulse $\delta(t))$ is visualized by Figure $4\left(p_{2}\right)$ :

Figure 4

Visualization of the idealized Delta-impulse

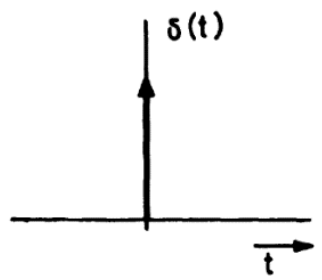

The third important property of the Delta-impulse, the sifting property $\int_{-\infty}^{\infty} \varphi(t) \delta\left(t-t_{0}\right)=\varphi\left(t_{0}\right)$, is deduced as follows: $\delta(t)$ is replaced by a function series $\left\{f_{n}\right\}$ $\left(p_{1}\right)$ according to Figure $3\left(p_{2}\right)$.For narrower and narrower pulses $f_{n}\left(t-t_{0}\right)$ the function 
$\varphi(t)$ could be replaced by the value $\left(t_{0}\right)\left(p_{4}\right)$. Using property (ii) $\left(p_{5}\right)$ and referencing to Figure $5\left(p_{2}\right)$, the sifting property follows.

Figure 5

\section{Sifting property}

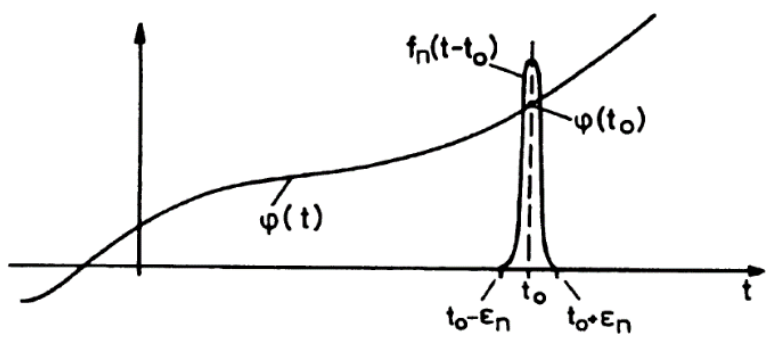

The technological-theoretical discourse is especially based on the general principle connecting idealized and real signals, illustrated in Figure 1. This provides justifications for $p_{1}$ and $p_{3}\left(l_{1}\right)$ : The definition via a series of real impulses reflects the interplay between real and idealized signals. Furthermore, it is argued that pulsed signals are very useful for engineering $\left(l_{2}\right)$ (p. 12). The properties (i) and (ii) are directly linked to properties of pulsed signals: only the action of the signal matters, not the specific form $\left(l_{2}\right)$. This is fulfilled, if the duration of the signal is very short, i.e. $2 \epsilon$. The action of the signal corresponds to the area, in Fettweis also denoted by "Impulsmoment", illustrated in Figure 6. This refers to the idea of the integral as area from mathematics courses $\left(l_{4}\right)$.

Figure 6

Example of a real impulse

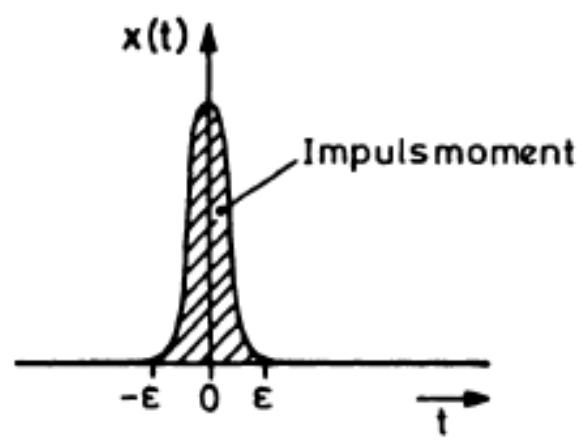


The element $p_{2}$ is produced by the justifying characteristic of visualizations $\left(l_{5}\right)$ : In Figure 3 a limit-process of real signals is visualized and the corresponding result is shown in Figure 4. So the visualizations reflect also the idealization process. The illustrations act as ostensive metaphors. Dirac (1963) claimed: "The delta function comes in just from picturing the infinity as something, which approximates to them." Additionally the principle, that the exact course of the signal doesn't matter, the essential is, that it is pulsed is important for all figures $\left(l_{3}\right)$. The ever shorter durations of the pulsed signals justify the replacement of $\varphi(t)$ by $\left(t_{0}\right)\left(l_{6}\right)$. The properties a) and b), which are assigned in $p_{3}$, are idealizations of properties (i) and (ii), properties of real signals, which are transferred to properties of an ideal signal. Especially property b), which contradicts the understanding of the integral in higher mathematics courses, is not mathematically justified yet. Fettweis (p. 14) discusses this point explicitly and justifies the integration by referring to real signals.

Summarizing, the technological-theoretical discourse is a mixture of higher mathematics ideas $\left(l_{4}\right)$, engineering reasoning (i.e $l_{2}$ and $l_{3}$ ) and a principle concerning the interplay between real and idealized signals reflecting the connection of mathematics and physics in general $\left(l_{1}\right)$. The justification and explanation of practices considering idealized signals like $\delta(t)$ are done on the level of real signals. This correlates with Dirac's (1958) hint, that one must exit the mathematical context for justification and do not interpret $\delta$ as mathematical symbol.

The reconstructed elements of the logos block and other justifications and explanations could be assigned to different levels of the scale of levels of codetermination: The justification of the principle in Figure 1 lies on the level of the discipline (physics). The technological-theoretical elements $l_{1}, l_{2}, l_{4}$ and $l_{5}$ could be assigned to domain (engineering), $l_{3}$ to sector (signal theory) and $l_{6}$ to the level of the 
subject (Delta-impulse). The local curriculum (level of university) and experience and propensity of the author of the textbook are also mentioned as a restriction for the content (Fettweis, p. iii). Finally we refer to a remark by Peters $(2004$, p. 99) that adapts a statement by Schwinger about Feynman-diagrams: "the $\delta$-function 'was bringing computation to the masses"“, which expresses the teaching and learning process related institutional aspect of the Dirac-impulse in a rather convincing way. Moreover, this statement indicates aspects on the level of society.

\section{Further remarks related to epistemological-philosophical issues}

The Dirac-impulse represents an idealized signal. Via approximation sequences this idealized signal, which is neither observable nor measurable, was linked to real signals, which are in principle observable and measurable. The scheme in figure 1 represented the basic consideration that underlies specific and, regarding the SST-context, adequate identifications. In particular theoretical relations including $\delta$ as well as $\delta$ itself gain empirical meaning: idealized signals like $\delta$ become physical quantities in the sense of 3.2, which allows formulating relations like the sifting property and measurements. These interrelations (e.g. allowing measurement, being element of a relation) imprint certain properties and induce techniques and technologies treating $\delta$, which look purely mathematically and were historically important aspects for developing a systematic and axiomatic based mathematics for $\delta$. From the physical point of view this might be helpful but is not necessary.

Furthermore, the mathematical theory as such does not allow injecting into $\delta$ physical meaning how it is enabled by, among others, the scheme in figure 1: For linking $\delta$ with measurable quantities, it has occasionally to be replaced in a SST adequate way, which necessarily transcends the formal mathematical context. In particular the inherent 
and specific identification of "finite" and "infinite" cannot mathematically be proved to be correct but could only be mathematically explored.

Moreover, the sifting property links a global continuous object $\varphi$ to local values $\varphi\left(t_{0}\right)$. This is one of the issues of $\delta$ in equations, e.g. in transferring relations from discrete signals to continuous signals and vice versa. This gives the possibility for treating the dialectic between "point" and "continuum" in such a way that allows computation $(\delta$ appears in equations and calculus) and measuring.

\section{Conclusion}

We claim that a broad understanding of logos-blocks in praxeological reference models taking into account higher levels of codetermination is valuable, since it allows in particular identifying inherent issues, which have to be resolved in some way by any institutionalized didactical or pedagogical practice. Here the aim was amongst others to illustrate and identify the relevance of basic philosophical-epistemological ideas for enriching the logos-block of praxeologies in SST and how they contribute to a wider understanding of actual justifications of practices. We will move on in this direction.

\section{References}

Alpers, B. Differences between the usage of mathematical concepts in engineering statics and engineering mathematics education. In: Didactics of Mathematics in Higher Education as a Scientific Discipline - Conference Proceedings, khdm-Report 1705, Kassel: Universität Kassel, p. 137-141, 2017.

Antosik, P., Mikusiński, J., \& Sikorski, R. Theory of distributions: the sequential approach. Amsterdam [u.a.]: Elsevier Scientific Publication, 1973.

Barquero, B., Bosch, M., \& Gascón, J. 'Applicationism' as the dominant epistemology at university. In: CERME 7-Seventh Congress of the European Society for Research in Mathematics Education, p. 1937-1948, 2011.

Barquero, B., Serrano, L., \& Serrano V. Creating the necessary conditions for mathematical modelling at university. In: CERME8-Eight Congress of the European Society For Research In Mathematics Education, p. 950-959, 2013.

Blum, W., \& Leiss, D. How do students and teachers deal with mathematical modelling problems? The example "Sugarloaf". ICTMA 12 Proceedings, p. 222-231, 2005. 
Bosch, M., \& Gascón, J. Twenty-five years of the didactic transposition. ICMI Bulletin, 58, p. 51-65, 2006.

Borzeszkowski, H. H. V., \& Wahsner, R. Das physikalische Prinzip: der epistemologische Status physikalischer Weltbetrachtung. Königshausen \& Neumann, 2012.

Castela, C., \& Romo Vázquez, A. Des mathématiques à l'automatique : étude des effets de transposition sur la transformée de Laplace dans la formation des ingénieurs. Recherches en Didactique des Mathématiques, 31(1), p. 79-130, 2011.

Chevallard, Y. Fundamental concepts in didactics: Perspectives provided by an anthropological approach. Recherches en Didactique des Mathématiques Selected Papers. La Pensée Sauvage, Grenoble, p. 131-167, 1992.

Chevallard, Y. L'analyse des pratiques enseignantes en théorie anthropologique du didactique. Recherches en Didactique des Mathématiques 19(2), p. 221-266, 1999.

Chevallard, Y., BOSCH, M., \& KIM, S. What is a theory according to the anthropological theory of the didactic? In: CERME 9-Ninth Congress of the European Society for Research in Mathematics Education, p. 2614-2620, 2015.

Dirac, P. A. The physical interpretation of the quantum dynamics. Proceedings of the Royal Society of London. Series A, Containing Papers of a Mathematical and Physical Character, 113(765), p. 621-641, 1927.

Dirac, P.A.M. (1958). The principles of quantum mechanics. 4. ed. Oxford: Clarendon Press, 1958.

Dirac, P.A.M. Interview with T.S. Kuhn. Archives for the History of Quantum Physics, Niels Bohr Library, AIP, New York, 1963.

Fettweis, A. Elemente nachrichtentechnischer Systeme. Wiesbaden: Vieweg \& Teubner Verlag, 1966.

Girod, B., Rabenstein, R., \& Stenger, A. K. E. Einführung in die Systemtheorie - Signale und Syteme in der Elektrotechnik und Informationstechnik. Wiesbaden: B.G. Teubner Verlag, 2007.

Hochmuth, R., Biehler, R., \& Schreiber, S. Considering mathematical practices in engineering contexts focusing on signal analysis. Proceedings of RUME17, p. 693-699, 2014.

Hochmuth, R. \& Schreiber, S. Conceptualizing Societal Aspects of Mathematics in Signal Analysis. In: Proceedings of the Eight International Mathematics Education and Society Conference Vol. 2, Portland: Ooligan Press, p. 610-622, 2015.

Landers, D. \& Rogge, L. Nichtstandard Analysis. Springer-Verlag, 2013.

Peters, K. H. Der Zusammenhang von Mathematik und Physik am Beispiel der Geschichte der Distributionen: Eine historische Untersuchung über die Grundlagen der Physik im Grenzbereich zu Mathematik, Philosophie und Kunst. Dissertation, Universität Hamburg, 2004.

Peters, J., Hochmuth, R., \& Schreiber, S. Applying an extended praxeological ATDModel for analyzing different mathematical discourses in higher engineering courses. In: Didactics of Mathematics in Higher Education as a Scientific 
Discipline - Conference Proceedings. khdm-Report 17-05 (pp. 172-178). Kassel: Universität Kassel, 2017.

Purkert, W. Infinitesimalrechnung für Ingenieure-Kontroversen im 19. Jahrhundert. In: Rechnen mit dem Unendlichen, Basel: Birkhäuser, p. 179-192, 1990.

Schwartz, L. Théorie des distributions et transformation de Fourier. In: Annales de l'Université de Grenoble, Vol. 23, p. 7-24, 1947.

Tuminaro, J., \& Redish, E. F. Elements of a cognitive model of physics problem solving: Epistemic games. Physics Education Research, 3, p. 1-22, 2007.

Wahsner, R., \& Borzeszkowski Von, H.-H. Die Wirklichkeit der Physik. Studien zur Idealität und Realiät in einer messenden Wissenschaft. Frankfurt/M., Berlin, Berlin: Peter Lang, 1992.

Weisbach, J. L. Die ersten Grundlehren der höhern Analysis oder der Differenzial-und Integralrechnung: Für das Studium der praktischen Mechanik und Naturlehre möglichst populär. Braunschweig: Vieweg und Sohn, 1860. 